\title{
VALIDACIÓN DE LA ENCUESTA DE CALIDAD Y SATISFACCIÓN DE SIMULACIÓN CLIINICA EN ESTUDIANTES DE ENFERMERÍA
}

\section{VALIDATION OF QUALITY AND SATISFACTION SURVEY OF CLINICAL SIMULATION IN NURSING STUDENTS}

\author{
Ángela Astudillo Araya * \\ Miguel Ángel López EsPinOzA ** \\ Víctor CÁdiz Medina ${ }^{* * *}$ \\ Jaqueline Fierro PaLma ${ }^{* * * *}$ \\ Andrea Figueroa Lara \\ NiCOLE VILCHES PARRA ${ }^{* * * * * x}$
}

\begin{abstract}
RESUMEN
La simulación clínica de alta fidelidad es considerada una metodología fundamental para la formación del estudiante. Una forma de evaluar la efectividad de esta metodología es por medio de la satisfacción de los estudiantes. Objetivo. Validar los constructos de la versión española de la escala "Encuesta de calidad y satisfacción de simulación clínica" de Durá Ros. Material y Método: Estudio transversal. La muestra fue de 216 estudiantes de $3^{\circ}$ a $5^{\circ}$ año de la carrera de Enfermería de la Universidad del Bío-Bío, Chillán-Chile. Se aplicó análisis de componentes principales con rotación Varimax. La consistencia interna se evaluó con $\alpha$-Cronbach. Resultados: De los 216 estudiantes, el $37 \%$ cursaban el $3^{\circ}$ año, el $36,6 \% 4^{\circ}$ año y el $26,4 \%$ el $5^{\circ}$ año de la carrera. El $87,5 \%$ ha obtenido una calificación promedio acumulada de 5,0-5,99 (escala de 1-7). Se obtuvo un coeficiente $\mathrm{KMO}=0,887$, y la prueba de esfericidad de Bartlett fue estadísticamente significativa $\left(\chi^{2}=1014,977 ; 105 \mathrm{gl} ; \mathrm{p}<\right.$ 0,001), el análisis de componentes principales (ACP) resultó en tres componentes que visualizan los conceptos: aprendizaje significativo, estructura de la simulación clínica y relación interpersonal con la simulación clínica, los que en conjunto lograron explicar un $51,277 \%$ de la varianza total. El coeficiente alfa de Cronbach para los componentes fue $\alpha=0,865 ; 0,467$ y 0,550 respectivamente. Conclusión. Emergieron tres componentes que caracterizan la satisfacción de los estudiantes con respecto a la simulación clínica. Se sugiere continuar con el trabajo exploratorio, hasta llegar a un análisis factorial confirmatorio que permita evaluar la bondad de ajuste del modelo con que se valida la escala.
\end{abstract}

Palabras clave: Satisfacción personal, entrenamiento simulado, estudiantes de enfermería, estudios de validación.

\footnotetext{
*Enfermera. Magíster en Pedagogía para la Educación Superior. Académica U. del Bío-Bío. Chillán, Chile. Email: aastudil@ ubiobio.cl

${ }^{* *}$ Nutricionista. Máster en Metodología de la Investigación Cs de la Salud. U. de Salamanca. España. Email: milopez@usal.es *** Enfermero. Departamento de Salud Municipal (DESAMU) comuna del Carmen. Chillán, Chile. Email: vcadizm@gmail. com

${ }^{* * * *}$ Enfermera. Hospital Regional de Concepción Guillermo Grant Benavente. Concepción, Chile. Email: j.morelia91@gmail. com

****** Enfermera Cuidados medios Médicos-Quirúrgicos Hospital Clínico Herminda Martin. Chillán, Chile. Email: Melinda. figueroa.lara@gmail.com

${ }_{* x+x+* *}^{*}$ Enfermera TIM Pediátrico Hospital Herminda Martin. Chillán, Chile. Email: nicole.emp.vilches.parra@gmail.com
} 


\section{ABSTRACT}

High-fidelity clinical simulations are considered a fundamental method of training for nursery students. One way of assessing the effectiveness of this method is through student's satisfaction. Objective: To validate the constructs of the Spanish version of the scale presented in "Survey of Quality and Satisfaction of clinical simulation" by Durá Ros. Method: A cross-sectional study was used, with a sample consisting of 216 undergraduate nursing students from third to fifth year from the University of Bío-Bío, in Chillán, Chile. A Principal Components Analysis (PCA) with Varimax rotation was used. Internal consistency was evaluated with $\alpha$-Cronbach. Results: Of the 216 students, $37 \%$ were in the third year of the program, $36.6 \%$ in the fourth year and $26.4 \%$ in the fifth year. Using a grading scale of 1 to $7,87.5 \%$ of students averaged grades between 5.0 and 5.99 . A KMO $=0.887$ coefficient was obtained, and the Bartlett's sphericity test was statistically significant $(\chi 2=1014,977 ; 105 \mathrm{gl} ; \mathrm{p}<$ 0.001). The Principal Components Analysis (PCA) resulted in three components: significant learning, structure of the clinical simulation and interpersonal relationship with the clinical simulation. Together, these components managed to explain $51.227 \%$ of the total variance, with $\alpha$ values being $0.865,0.467$ and 0.550 respectively. Conclusion: Three components emerged from the analysis, which help characterize student's satisfaction regarding clinical simulation. Exploratory work is suggested until a confirmatory factor analysis is reached to evaluate the goodness of fit of the model.

Key words: Personal satisfaction, simulation training, students, nursing, validation studies.

Fecha recepción: 21/12/15 Fecha aceptación: 18/11/16

\section{INTRODUCCIÓN}

La formación del profesional de enfermería debe estar centrada en el estudiante, con profesores calificados en metodologías activas, en donde el docente es un facilitador del aprendizaje y en su práctica docente debe incorporar diferentes formas de evaluar y asegurar el cumplimiento de las metas u objetivos planteados en los perfiles de egreso ${ }^{1}$.

En la actualidad, una de esas estrategias didácticas de enseñanza-aprendizaje es la simulación clínica, en donde las experiencias guiadas replican aspectos sustanciales del mundo real (1). Específicamente, la simulación clínica en enfermería, alrededor del mundo, surge como un método para aprender y valorar conocimientos, habilidades téc-

${ }^{1}$ Velasco Martín A. Simulación clínica y enfermería: creando un ambiente de simulación. [Trabajo fin de Grado en Enfermería]. Santander: Universidad de Cantabria; 2013. [citado 26 abril 2016]. 36 p. Disponible en: http://repositorio.unican.es/xmlui/bitstream/handle/10902/3949/VelascoMartinA.pdf? sequence $=1$ \&isAllowed $=\mathrm{y}$ nicas o no técnicas y actitudes, en el ámbito de las ciencias de la salud $(2,3)$. A la vez, se convierte en un método de entrenamiento efectivo, pues ofrece al estudiante la oportunidad de realizar una práctica similar a la que desarrollara en su posterior práctica clínica.

Asumiendo estas ventajas, ha parecido importante validar un instrumento que permita medir, en un ambiente clínico, el grado de satisfacción de los receptores de este método pedagógico, en este caso estudiantes de Enfermería.

La satisfacción, en el contexto de una práctica clínica competente, se explica por tres constructos: "debriefing y reflexión", "razonamiento clínico" y "aprendizaje clínico" (4). El debriefing es la etapa clave de la simulación, corresponde al tiempo otorgado para el análisis sistemático del escenario, lo que resultó bien o no tan bien. El instructor guía la autorreflexión de los participantes y los complementa con comentarios, es decir, no solo se requiere de habilidades procedimentales y de conocimiento, sino también de habilidades de razonamiento clínico, que es 
la capacidad de analizar, sintetizar y tomar decisiones.

Precisamente, la simulación clínica permite que los estudiantes puedan poner en práctica estos constructos con la experimentación de un caso clínico simulado. Lo anterior se convierte en un aprendizaje significativo trascendental, definido como "el mecanismo humano por excelencia para adquirir y almacenar la vasta cantidad de ideas e información representada por cualquier campo del conocimiento", y que se desarrolla al relacionar la información previa del estudiante con la que es adquirida más recientemente y es en este proceso en donde se reajustan y reconstruyen los conocimientos (5).

Existen dos consecuencias trascendentales para este proceso de aprendizaje: la primera es que el periodo de retención de la información es mayor y la segunda es que los conocimientos recientes se incorporan a los ya existentes, es decir, se realiza una interacción del nuevo aprendizaje con la noción de conocimiento preexistente en el individuo (6). $\mathrm{Al}$ inicio, estos conocimientos se encuentran disociados por conocimientos anteriores que el individuo posee y que están previamente organizados y jerarquizados. Posterior a ello, los nuevos conocimientos se van incorporando progresivamente a la organización ya establecida, generando la asociación completa de un solo conocimiento $(7,8)$. Así, la enseñanza es más efectiva, pues al estudiante se le da la oportunidad de elegir su propio proceso al presentarle problemas que debe resolver, a la vez que debe formular sus propias preguntas, con el propósito de que proyecte sus objetivos de conocimiento y pueda observar sus logros (9).

La teoría del aprendizaje es la que más se conecta con la simulación clínica, en donde se puede observar un aprendizaje concebido en tres fases sucesivas: primero, una experiencia concreta, es decir, la interacción primaria con el objeto en estudio a fin de conocerlo en forma concreta y vivencial; segundo, la observación reflexiva, que permite extraer ideas y pensar sobre ello, es decir, reflexionar sobre lo que se ha realizado para poder generar aprendizaje y tercero, la conceptualización abstracta y experimentación activa, es decir, contrastar lo observado con la información y explicaciones del profesor (10). Todo lo aprendido en estas fases se pone en práctica y se transfieren a otros contextos, potenciándolo para generar un aprendizaje duradero y útil en el futuro, que es lo que experimentan los estudiantes al someterse a la simulación de alta fidelidad, en donde van asimilando los conocimientos previos con la nueva información entregada en la simulación.

Precisamente, este aprendizaje significativo adquirido durante la simulación clínica toma valor en el debriefing cuando, posterior al escenario simulado, se logra un espacio de reflexión guiado por el docente, quien favorece el aprendizaje reflexivo y significativo de la experiencia vivida, da espacio para la autoevaluación, refuerza las buenas prácticas y el espíritu de trabajo en equipo (9), en otras palabras, un aprendizaje experiencial, que enfatiza la importancia que tiene experimentar ciertas situaciones para aprenderlas (10).

Según lo descrito y asumiendo el supuesto de que la satisfacción está asociada a una mayor motivación a aprender por parte de los estudiantes y que es crucial para evaluar la enseñanza, a los docentes y a los propios estudiantes, es necesario contar con instrumentos que describan el nivel de satisfacción respecto a la metodología de simulación clínica.

Por su utilidad para la práctica clínica de enfermería, el objetivo de este estudio fue validar la versión española de la escala "Encuesta de calidad y satisfacción de simulación clínica" de Durá, quién en 1998, la adaptó de una encuesta creada en la Universidad de Harvard (USA), mediante técnica de panel de expertos, para describir la satisfacción de los estudiantes con la simulación clínica de alta fidelidad, que es un tipo de simulación diseñada para la adquisición de competencias avanzadas y la resolución de casos clíni- 
cos, en donde se recrean espacios de simulación realistas con simuladores de pacientes humanos (11). El cuestionario consta de 15 ítems con un diseño unifactorial relacionados con el aprendizaje mediante simulación clínica de alta fidelidad como herramienta docente más un apartado de observaciones con respuesta abierta.

Desde el año 2006 ha sido aplicada a aproximadamente 4.000 alumnos españoles. La validación del instrumento se fundamenta en el número de encuestas aplicadas, presentando validez de contenido, siendo modificada y adaptada mediante técnica de panel de expertos (12), sin embargo, no presenta propiedades psicométricas aplicadas en Chile.

\section{MATERIAL Y MÉTODO}

Tipo de estudio: Estudio de abordaje cuantitativo, transversal de validación local.

Población y muestra: El Universo estuvo constituido por 350 estudiantes de la Carrera de Enfermería de la Universidad del Bío-Bío, Chile. Para seleccionar la muestra se aplicaron los siguientes criterios de inclusión: estudiantes matriculados en el periodo 20132014, que hayan participado en al menos una sesión de simulación clínica de alta fidelidad y haber cursado, al menos, un periodo de prácticas clínicas. Se excluyeron estudiantes que ingresaron por medio de traslado desde carreras de Enfermería de otras casas de estudios, con el objetivo de mantener una muestra homogénea en términos de las técnicas educativas que se implementan para la adquisición del conocimiento. Finalmente participaron 216 estudiantes de pregrado, de $3^{\circ}$ a $5^{\circ}$ año.

Recolección de datos: La escala "Encuesta de calidad y satisfacción de simulación clínica" consta de 15 ítems con cinco posibilidades de respuestas, puntuadas de 1 (muy en desacuerdo) a 5 (muy de acuerdo), y un último ítem para observaciones. Presenta un diseño original unifactorial que no ha sido testeado por pruebas psicométricas, pero con validez de contenido (panel de expertos). Ha sido aplicada a 4.000 alumnos españoles, quienes realizaron diversas sugerencias, adaptándola a la realidad cultural española ${ }^{2}$. La evaluación de los sujetos se realiza de acuerdo a la sumatoria de puntajes obtenidos, cuyo máximo, de 75 puntos, se interpreta como mayor grado de satisfacción y el mínimo, de 15 puntos, menor grado de satisfacción. Para estimar el tiempo de respuesta y valorar la comprensión del instrumento, se realizó una prueba piloto, en el año 2014, con 10 estudiantes de $4^{\circ}$ año, de la misma carrera, impartida en una Universidad de una ciudad cercana, que posee una metodología de simulación similar a la desarrollada en la Universidad del Bío-Bío. Por muestreo no probabilístico accidental, se invitó a contestar la escala a los estudiantes que se encontraban en el aula de simulación en un día y hora específicos. Se concluyó que el instrumento fue comprendido por los estudiantes, por lo que no se realizaron modificaciones de la versión original.

Consideraciones éticas: El estudio fue autorizado por las autoridades académicas y administrativas de la Escuela de Enfermería de la Universidad del Bío-Bío y los estudiantes que participaron fueron aquellos que dieron su consentimiento informado, siguiendo los principios éticos para las investigaciones biomédicas (13-15) y la legislación vigente en Chile (16). Se hizo hincapié en el documento que la participación en el estudio era de carácter voluntario, confidencial y que no perseguía medir conocimiento ni condicionaba de modo alguno la relación del estudiante con la evaluación académica o la relación con sus docentes. El levantamiento de los datos se realizó en las aulas de clases entre abril y mayo de 2014, sin la presencia del do-

\footnotetext{
${ }^{2}$ Cádiz Medina V. Satisfacción con la simulación clínica como herramienta docente para el aprendizaje en estudiantes de Enfermería de las Universidades de Alicante (España) y Bío-Bío (Chile). [Tesis fin de grado Enfermería]. Chillán, Chile: Universidad del Bío-Bío; 2014. p. 102.
} 
cente a cargo de la cátedra para evitar potencial influencia en las respuestas. Fue aplicado por un equipo de estudiantes de último año de la carrera previamente capacitados con el objetivo de estandarizar el procedimiento de entrega de cuestionarios y orientar potenciales dudas con el llenado del cuestionario sin influenciar las respuestas de la muestra. El tiempo de aplicación del instrumento fue, en promedio, de $15 \mathrm{~min}$.

Análisis de datos: En el análisis estadístico, las variables de naturaleza cualitativa se describieron con frecuencias observadas y porcentajes; para las variables cuantitativas se aplicaron medias y desviaciones típicas. Para reducir los reactivos de la encuesta en dimensiones, se desarrolló un análisis de Componentes Principales (dado que es primera vez que se aplica en una muestra a nivel local), con rotación Varimax, previa comprobación de pertinencia con un coeficiente de Kaiser-Meyer-Olkin (KMO) mayor a 0,50 y prueba de esfericidad de Bartlett estadísticamente significativa a nivel de 0,05 . Los componentes se construyeron teniendo en cuenta una saturación o autovalor mayor a 1,0. La fiabilidad de la escala y de los componentes extraídos, luego de la solución factorial rotada, se evaluó con el coeficiente alpha $(\alpha)$ de Cronbach. Los datos fueron procesados en el programa estadístico SPSS, versión 15 (en español).

\section{RESULTADOS}

El mayor porcentaje de estudiantes se encontraba en un rango etario entre 19 y 22 años; existió un predominio del sexo femenino; en cuanto al lugar de procedencia del estudiante, la tendencia es clara hacia la residencia en la misma ciudad donde se emplaza la casa de estudio. Respecto a las características académicas, las mayores proporciones se comparten entre tercero y cuarto (37 y 36,6\% respectivamente). En cuanto al rendimiento académico, las calificaciones de los estudiantes se concentraron en el rango bueno $(5,0 \mathrm{a}$ 5,99 ) con un $87,5 \%$; mientras que en el rango suficiente se observó un 10,6\% de frecuencia (Tabla 1).

La Tabla 2 describe los puntajes medios de los 15 ítems de la "Encuesta de calidad y satisfacción de simulación clínica". En los promedios de todos los ítems no se observan puntajes menores a 3, lo que sugiere que los estudiantes no estuvieron en desacuerdo con ningún ítem. El máximo puntaje obtenido se relacionó con la utilidad de la simulación clínica para el aprendizaje de los estudiantes (4,7 DS 0,6). Los menores puntajes medios fueron para la pregunta referente a la duración de la sesión de simulación clínica $(3,4$ DS 1,2). En relación al ítem "En general, la experiencia con simulación clínica ha sido satisfactoria", la tendencia fue positiva $(4,1$ DS 0,8 ), lo que muestra que los estudiantes cumplieron sus expectativas al experimentar la simulación clínica de alta fidelidad.

Respecto a la factibilidad de un análisis factorial exploratorio, mediante componentes principales, el coeficiente KMO mostró un valor de 0,887 y la prueba de esfericidad de Bartlett fue estadísticamente significativa $\left(\chi^{2}=1014,977 ; 105 \mathrm{gl} ; \mathrm{p}<0,001\right)$, lo cual sugirió la conformación de reactivos en componentes.

La Tabla 3 presenta el análisis de componentes principales, del cual fue posible extraer 3 componentes, que en conjunto lograron explicar un 51,277\% de la varianza total. El coeficiente alfa de Cronbach global fue de $\alpha=0,861$. El primer componente presentó un $\alpha=0,865$; el segundo, $\alpha=0,467$, y el tercero, de un $\alpha=0,550$.

El componente 1 agrupó lo que se denominó "aprendizaje significativo", debido a que integra ítems relacionados con la formación de conocimientos sólidos, basados en los saberes previos del estudiante; está compuesto por 7 (ítems 1, 3, 5, 6, 7, 14, 15) de los 15 ítems del instrumento original, quienes explican $27,89 \%$ de la varianza total. El com- 
Tabla 1. Distribución de los estudiantes de Enfermería según características sociodemográficas y académicas.

\begin{tabular}{lrc}
\hline \multirow{2}{*}{ Características sociodemográficas y académicas } & \multicolumn{2}{c}{ Chile $(\mathbf{n}=\mathbf{2 1 6})$} \\
\cline { 2 - 3 } Edad & & $\%$ \\
$\quad$ Entre 19 y 22 años & 158 & 73,1 \\
$\quad$ Entre 23 y 26 años & 50 & 23,1 \\
$\quad 27$ años y más & 8 & 3,7 \\
Sexo $\quad$ & 158 & 73,1 \\
$\quad$ Mujer & 58 & 26,9 \\
$\quad$ Hombre & & \\
Lugar de Procedencia & 187 & 86,6 \\
$\quad$ Urbano & 29 & 13,4 \\
$\quad$ Rural & & \\
Nivel cursado & 80 & 37 \\
$\quad$ Tercero & 79 & 36,6 \\
$\quad$ Cuarto & 57 & 26,4 \\
$\quad$ Quinto & & \\
Rendimiento académico $\left.{ }^{*}\right)$ & 23 & 10,6 \\
$\quad$ Suficiente & 189 & 87,5 \\
$\quad$ Bueno & 4 & 1,9 \\
$\quad$ Muy bueno
\end{tabular}

${ }^{\star}$ ) Siendo suficiente: 4,00-4,99; bueno: 5,00-5,99; muy bueno: 6,00-6,99

Tabla 2. Puntajes medios de los ítems de la "Encuesta de calidad y satisfacción de simulación clínica” (versión original, previo al Análisis Factorial Exploratorio).

Ítems

Chile Media \pm DS

1. La simulación es un método docente útil para el aprendizaje $4,7 \pm 0,6$

2. Los escenarios donde se desarrolla la simulación son realistas $3,5 \pm 0,9$

3. La experiencia con simulación ha mejorado mis habilidades técnicas

$4,1 \pm 0,7$

4. La simulación ayuda a desarrollar el razonamiento crítico y la toma de decisiones

$4,1 \pm 0,8$

5. Los casos simulados se adaptan a mis conocimientos teóricos

$4,2 \pm 0,7$

6. La experiencia con el simulador ha aumentado mi seguridad y confianza

$4,0 \pm 0,8$

7. La simulación me ha ayudado a integrar teoría y práctica

$4,2 \pm 0,7$

8. Los talleres con el simulador me han motivado a aprender

$4,0 \pm 0,8$

9. En simulación, es útil el ver las propias actuaciones grabadas

$3,8 \pm 1,0$

10. La duración del caso es adecuada

$3,4 \pm 1,2$

11. La capacitación del profesorado es adecuada

$4,0 \pm 0,8$

12. La simulación fomenta la comunicación entre los miembros del equipo

$4,3 \pm 0,7$

13. La simulación clínica ayuda a priorizar actuaciones de Enfermería

$4,2 \pm 0,7$

14. La interacción con la simulación ha mejorado mi competencia clínica

$4,1 \pm 0,8$

15. En general, la experiencia con simulación clínica ha sido satisfactoria.

$4,1 \pm 0,8$ 
Tabla 3. Análisis Factorial Exploratorio de la versión original ( 15 ítems) del cuestionario Calidad y Satisfacción con la Simulación Clínica aplicada a estudiantes de Enfermería chilenos $(\mathrm{n}=216)$.

Items (reactivos)

. La simulación me ha ayudado a integrar teoría y práctica

6. La experiencia con el simulador ha aumentado mi seguridad y confianza

14. La interacción con la simulación ha mejorado mi competencia clínica

3. La experiencia con simulación ha mejorado mis habilidades técnicas

15. En general, la experiencia con simulación clínica ha sido satisfactoria

5. Los casos simulados se adaptan a mis conocimientos teóricos

1. La simulación es un método docente útil para el aprendizaje

4. La simulación ayuda a desarrollar el razonamiento crítico y la toma de decisiones

13. La simulación clínica ayuda a priorizar actuaciones de Enfermería

2. Los escenarios donde se desarrolla la simulación son realistas

8. Los talleres con el simulador me han motivado a aprender

9. En simulación, es útil el ver las propias actuaciones grabadas

10. La duración del caso es adecuada

11. La capacitación del profesorado es adecuada

12. La simulación fomenta la comunicación entre los miembros del equipo

Varianza total explicada

Autovalor del factor

Alpha de Cronbach

\begin{tabular}{cccc}
\multicolumn{3}{c}{ Componentes } & \\
\cline { 1 - 3 } & 2 & 3 & \\
& & 2 &
\end{tabular}

0,719

0,567

0,697

0,571

$\mathbf{0 , 6 5 3} \quad 0,352$

0,588

0,651

0,465

$\mathbf{0 , 6 3 7} \quad 0,374$

0,579

0,588

0,396

0,585

0,425

$0,566 \quad 0,308$

0,417

0,523

0,301

0,333

0,475

$0,506 \quad 0,342$

0,377

$0,414 \quad 0,402$

0,37

0,846

0,719

0,457

0,337

0,41

0,842

0,73

0,354

0,689

0,603 ponente 2 presentó reactivos relacionados con la "estructura de la sesión de simulación clínica”, ya que señala aspectos relevantes de la constitución del caso por este método de enseñanza. Está integrado por 2 ítems $(9,10)$ que explican el $12,266 \%$ de la varianza. El componente 3 fue denominado "relación interpersonal en la simulación clínica”, debido 
a que describe la interacción que produce la actividad de simulación entre los estudiantes y el docente a cargo, todos como un equipo de trabajo. Está compuesto por 2 ítems (11, 12) y explican el $11,12 \%$ del total de la varianza.

\section{DISCUSIÓN Y CONCLUSIÓN}

A modo descriptivo, al aplicar la versión original de la "Encuesta de calidad y satisfacción de simulación clínica” se presentaron puntajes medios mayores a 3 en los 15 ítems (reactivos), lo que refleja que no estuvieron en desacuerdo frente a ningún enunciado propuesto en la encuesta, observándose la puntuación máxima en el ítem 1 "La simulación clínica es un método docente útil para el aprendizaje", lo que coincide con el estudio de Durá realizado el año 2012 (3) el cual, basado en una muestra de 72 estudiantes de $2^{\circ}$ grado de la Universidad de Cantabria, encontró un evidente reconocimiento de la utilidad que confiere esta herramienta en el proceso educativo de los estudiantes, idea que se reafirma en el estudio realizado en la misma Universidad por Riancho et al., el cual, dentro de sus resultados obtenidos en las encuestas, concluyó que la actividad es valorada como muy positiva por los alumnos, quienes la encontraron "útil" con puntaje de 4,8 y 4,9 e "interesante" con una puntuación fluctuante de 4,9 (17). Las menores puntuaciones, en el presente estudio, se presentaron en el ítem "la duración del caso es adecuada", lo que se afirma en el apartado "observaciones" de la encuesta, en donde el alumnado señaló como un problema relevante el escaso tiempo de duración del caso simulado.

Con el fin de medir las propiedades psicométricas del instrumento, se aplicó un Análisis Factorial Exploratorio (AFE). Este método estadístico permitió evaluar la validez de constructo del instrumento "Encuesta de calidad y satisfacción de simulación clí- nica", encontrándose una estructura de tres componentes: "Aprendizaje significativo", "estructura de la sesión de simulación" y "relación interpersonal en la simulación clínica", distinta a la estructura unifactorial de la versión española. Sin embargo, es preciso señalar que la estructura unidimensional de la versión española no obedece al resultado de un análisis factorial, sino más bien obedece a la experiencia de la autora en relación a la simulación clínica de alta fidelidad, que a su vez está asociada a su propio contexto educativo y cultural, el cual puede afectar la comprensión de los ítems y posterior respuesta en los estudiantes chilenos.

Cabe señalar que otros estudios publicados han mostrado resultados diferentes en la validez factorial del instrumento. En un estudio australiano realizado por Levett-Jones et al., en una muestra de 268 estudiantes de Enfermería de segundo año y 76 de tercer año, en la Universidad de New Castle, el año 2011 (18), se realizó el proceso de validación de la encuesta para medir satisfacción con la simulación clínica de alta fidelidad mediante un análisis factorial exploratorio con rotación varimax. En base a esto, los autores lograron extraer estadísticamente tres componentes, estableciendo que el concepto "satisfacción" en esta muestra se explica a través de tres variables latentes: "debriefing y reflexión", "razonamiento clínico” " “aprendizaje clínico”; a diferencia de nuestra investigación en donde, si bien la encuesta presentó estructura trifactorial, los componentes fueron "aprendizaje significativo", "estructura de la sesión de simulación clínica” y "relación interpersonal en la simulación clínica”.

En el presente estudio, el primer componente denominado "aprendizaje significativo" definido por Ausubel como la incorporación de nueva información a la estructura cognitiva del estudiante, mediante interacción del nuevo aprendizaje con la noción de conocimientos preexistentes en el individuo (4) y ratificado por Kolb en su teoría del aprendizaje experiencial, la cual enfatiza la 
importancia que tiene experimentar ciertas situaciones para aprenderlas (9), está conformado por 7 ítems que hacen referencia de forma directa al "aprendizaje significativo", evidenciando la asimilación del conocimiento previo que el individuo poseía con la nueva información entregada en la simulación clínica de alta fidelidad.

El segundo componente denominado "estructura de la sesión de simulación clínica” en el cual son relevantes 2 aspectos abordados en los ítems "En simulación, es útil el ver las propias actuaciones grabadas" y "La duración del caso es adecuada”; según Durá, un punto importante de la estructura de la sesión de simulación es la duración del escenario que, aunque no presenta un tiempo establecido, es aconsejable que se desarrolle entre 15 y 20 min, además menciona que durante ese periodo de tiempo se debe realizar una videograbación de los participantes y, a su vez, el resto de los alumnos del grupo que no están participando pueden observarla en una sala contigua a través de pantallas de televisión (3). Así mismo, recomendaciones de la Universidad de Cádiz refieren que el "desarrollo del caso puede ser grabado en video, $y$ a la vez visualizado por el resto del grupo en el aula. Mientras están visualizando la actuación de los compañeros, cada alumno recoge en una plantilla los aspectos más relevantes que se están desarrollando relacionados con las habilidades técnicas y no técnicas" (19).

Los autores de este estudio nombraron al tercer componente "relación interpersonal en la simulación clínica”, el que integra los ítems "La capacitación del profesorado es adecuada" y "La simulación fomenta la comunicación entre los miembros del equipo". Jeffries et al. (20) plantean un modelo de simulación clínica, en donde interactúan 3 elementos inmersos en un ambiente de simulación: el estudiante, el profesor y las prácticas de enseñanza que se producen entre ellos; menciona que este último aspecto se ve influido por la relación que el estudiante presente con su docente durante la simulación clínica, dando como resultado de la interacción el aprendizaje y la satisfacción del alumno. Respecto a la comunicación dentro del equipo de trabajo, es necesario entender que las personas que lo integran son entes complejos en constante relación interpersonal, la que se define como una interacción recíproca entre dos o más personas; se trata de relaciones sociales en donde interviene la comunicación, que es la capacidad de las personas para obtener información respecto a su entorno y compartirla con el resto de la gente (21). Corvetto et al. mencionan en la revisión sistemática que "la educación y el entrenamiento basados en simulación han demostrado su efectividad en múltiples áreas. Específicamente ha demostrado mejorar la adquisición de conocimiento médico, la comunicación y el trabajo en equipo, el desarrollo de ciertas habilidades, disminuir el estrés durante los procedimientos e incluso ha mostrado directa mejoría de ciertos resultados clínicos" (1).

Los resultados del componente "aprendizaje significativo" muestran un alto grado de satisfacción por parte de los estudiantes con la simulación clínica como herramienta que potencia los conocimientos teóricos integrándolos a la práctica, fortalece las habilidades técnicas, aumenta la seguridad y confianza y mejora la competencia clínica del estudiante. Esto concuerda con el estudio de Alconero et al., en donde se señala la utilidad práctica de este método de enseñanza (22) (con un 87\% de estudiantes totalmente de acuerdo y 12\% de acuerdo), y la relación teoría y práctica que se genera a través de este (con un 80,3\% de alumnos totalmente de acuerdo y un 19,7\% de acuerdo). El estudio de Abdo y Ravert, de la Universidad de Brigham, Inglaterra (12), indica que los participantes de la simulación clínica recrean de mejor manera las situaciones de la realidad, logrando así estar preparados para una buena experiencia clínica, lo que conlleva a que presenten un aumento en el nivel de satisfacción, es decir beneficia la confianza para fortalecer el aprendizaje 
ocurrido en las aulas de clase.

En el componente "estructura de la sesión de simulación clínica", representó un grado de satisfacción que tiende a lo positivo, respecto a la utilidad de ver las propias actuaciones grabadas y a la duración del caso simulado. En el estudio de Durá (3) se menciona que la grabación y reproducción en vídeo de la actuación de los alumnos mejora no solo el aprendizaje sino también el comportamiento de los alumnos, y que debe utilizarse para dar a los participantes una perspectiva real de su actuación. Según el estudio de Valencia et al., llamado "Estandarización de procedimientos de Enfermería y producción de videos como estrategia de innovación académica", realizado en Chile el año 2013 (23), la técnica de videos educativos es una estrategia de enseñanza-aprendizaje efectiva que tiene alta valoración y satisfacción por parte de los estudiantes.

El componente "relación interpersonal en la simulación clínica" mostró un grado de satisfacción positivo ante el desarrollo de habilidades comunicativas tanto con el equipo como con el docente en la simulación clínica. Según el estudio de Alconero et al., los estudiantes indican que esta herramienta de aprendizaje mejora la comunicación con sus pares (con un 63,2\% de alumnos totalmente de acuerdo y un $28,9 \%$ de acuerdo) (22); se destaca además el establecimiento de prioridades y el desarrollo de habilidades para el trabajo en equipo logrado por los alumnos. En un estudio de Rojo y Díaz se menciona que la simulación como estrategia de enseñanza depende de la interacción del simulador, el objetivo y diseño de la actividad a simular y el rol docente desempeñado; de estos elementos, tres son de carácter educativo y dependientes del papel que el profesor realice en el proceso (6).

El promedio del puntaje global para la muestra representó una percepción positiva respecto al concepto de satisfacción con este método de enseñanza. Respecto a esto, se menciona en una investigación realizada por Bastías et al., que más del 50\% de los sujetos participantes del estudio están totalmente de acuerdo con que la simulación es un método útil para el aprendizaje, siendo de esta manera una metodología de enseñanza satisfactoria (24). Además, el estudio realizado por Achury et al. (25) señala en sus resultados que la satisfacción de los estudiantes frente a la simulación clínica fue en promedio 90,9\%, concluyendo este mismo, que los escenarios de simulación clínica de alta fidelidad se convierten en una herramienta novedosa y fundamental en el proceso de enseñanza- aprendizaje en Enfermería.

La satisfacción, cualquiera sea su contexto en la cual se aplique, es una variable difícil de medirla bajo el enfoque cuantitativo, es por ello que los instrumentos que intentan medirla deben sostener propiedades psicométricas aceptables, siendo el Análisis Factorial Exploratorio (AFE) una técnica estadística multivariante cuyo principal propósito es encontrar una manera de resumir la información contenida en una serie de variables originales a través de dimensiones compuestas (denominadas componentes) con una mínima pérdida de información, de manera que, en el caso de eliminar algún reactivo, no exista o haya escasa perturbación en los datos. Emergieron tres componentes que caracterizan la satisfacción de los estudiantes con respecto a la simulación clínica. Se sugiere aplicar métodos de análisis factorial confirmatorio para evaluar la bondad de ajuste del modelo con que se valida la escala ${ }^{3}$.

Se puede concluir que, de acuerdo al Análisis Factorial Exploratorio realizado en estudiantes chilenos, la satisfacción se explica por tres componentes: "aprendizaje significativo", "estructura de la sesión de simulación

${ }^{3}$ García Fuster A. Análisis de las características de diseño y ambientales de la Biblioteca de Ingeniería Industrial (UPV) y su influencia en la percepción de confort. [Proyecto fin de Grado en Ingeniería de Edificación].Valencia, España: Universidad Politécnica de Valencia; 2011. 
clínica” y "relación interpersonal en la simulación clínica”. Evidenciando un alto grado de satisfacción con la simulación clínica como herramienta que potencia los conocimientos previos del estudiante, favoreciendo el aprendizaje significativo, presentaron un grado de satisfacción tendiente a lo positivo respecto a la forma en que se organiza la sesión de simulación clínica y un alto grado de satisfacción ante el desarrollo de habilidades comunicativas y relación interpersonal con el docente y el equipo. Es importante señalar que existe escasez de instrumentos de medición de la satisfacción con la simulación clínica que se ajusten a la realidad nacional, por lo que es importante continuar el proceso de validación de la encuesta, aplicando métodos de análisis factorial confirmatorio para evaluar la bondad de ajuste del modelo con que se valida la escala.

Es importante señalar que este estudio involucra varias limitaciones, entre ellas está la escasez de teorías sobre satisfacción de los estudiantes con la simulación clínica de alta fidelidad que permitan sustentar los componentes que emergieron. Por otro lado, independiente de la cantidad muestral, este estudio está vinculado solamente a una Escuela de Enfermería, hecho que limita la validez externa de las conclusiones. Luego, a pesar que en el trabajo de campo se optó que la docente a cargo de la jornada de simulación no esté presente durante la aplicación del instrumento, puede que haya permeado las opciones de respuesta elegida. Además, la consistencia interna de dos componentes implica revisar la homogeneidad de los ítems que forman parte de ellos. Por estas razones este trabajo viene a animar a otros investigadores seguir explorando las propiedades psicométricas de este instrumento para que una vez que se clarifique la conformación de los componentes se pueda ejecutar análisis factorial confirmatorio con una muestra lo suficientemente representativa y evaluar la bondad de ajuste del modelo.

\section{REFERENCIAS}

1. Corvetto M, Bravo MP, Montaña R, Utili F, Escudero E, Boza C, et al. Simulación en educación médica: una sinopsis. Rev Med Chile. 2013; 141(1): 70-9.

2. Amaya AA. Elementos de las competencias: El ser, el saber y el saber hacer desarrollados a través de la simulación clínica en los estudiantes de medicina y ciencias de la salud. Rev Soc Col Oft [Internet]. 2006 [citado 26 abr 2016]; 39(3):12633. Disponible en: http://www.asepur.org/wp-content/uploads/2016/04/ Areas-del-Centro-de-Simulaci\%$\mathrm{C} 3 \% \mathrm{~B} 3 \mathrm{n}-\mathrm{Cl} \% \mathrm{C} 3 \% \mathrm{ADnica}-\mathrm{y}-\mathrm{elemen-}$ tos-de-las-competencias-1.pdf

3. Durá Ros MJ. La simulación clínica como metodología de aprendizaje y adquisición de competencias en Enfermería. [Tesis doctoral]. [Madrid]: Universidad Complutense de Madrid; 2013. 295 p.

4. Ausubel DP. Significado y aprendizaje significativo. En: Psicología educativa: un punto de vista cognoscitivo. México: Editorial Trillas; 1976. p. 53-106.

5. Asociación de Simuloeducadores de Puerto Rico. "Defiebring" y evaluación de la simulación [Internet]. 1er congreso ASEPUR más allá de la simulación; 27 oct 2012; Dorado, Puerto Rico; sf [citado 26 abr 2016]. 62 p. Disponible en http://www.asepur.org/wp-content/ uploads/2012/11/Debriefing-y-Evaluacion-en-la-Simulacion.pdf

6. Rojo Rojo A, Díaz Agea JL. El rol del instructor de simulación clínica: experiencia educativa en la UCAM. En: Muñoz Carril PC, Raposo Rivas M, González Sanmamed M, Martínez Figueira ME, Zabalza Cerdeiriña M, Pérez Abellás A, editores. Un practicum para la formación integral de los estudiantes [Internet]. XII Symposium internacional sobre el practicum y las prácticas en empresas; 26-28 jun 2013; 
Poio, España. Santiago de Compostela: Andavira; 2013. [citado 26 abr 2016]. p. 1485-1492. Disponible en https://www. academia.edu/5105592/EL_ROL_DEL_ INSTRUCTOR_DE_SIMULACION_ CLINICA._EXPERIENCIA_EDUCATIVA_EN_LA_UCAM

7. Utili Ramírez F. Simulación en el aprendizaje, práctica y certificación de las competencias en medicina. ARS médica. 2007; 36(2): 197-210.

8. Tomas U. El Psicoasesor. Teoría del Aprendizaje Significativo-David Ausubel [Internet]; 2011 Abr [citado 26 abr 2016]. Disponible en: http://elpsicoasesor.com/ teoria-del-aprendizaje-significativo-david-ausubel/

9. Kolb AD. Experiential learning: experience as the source of learning and development. Englewood Cliffs, NJ: Prentice Hall; 1984. 256 p.

10. Achury Saldaña D. Estrategias pedagógicas en la formación de profesionales de enfermería. Pontificia Universidad Javeriana [Internet]. 2008 [citado 26 abr 2016]; 10(2):101-111. Disponible en: http://file:///Users/joseleon/Downloads/1604-5126-1-PB.pdf

11. González MSG, Ramiro PAG. Evaluación de la calidad de dos modelos de simulación clínica. Opción [Internet]. 2016 [citado 4 jul 2017]; 32(11): 677-90 Disponible en: http://www.redalyc.org/ html/310/31048902039/

12. Abdo A, Ravert P. Student Satisfaction with Simulation Experiences. Clin Simul Nurs [Internet]. 2006 [citado 26 abr 2016]; 2(1): 13-6. Disponible en: http:// www.sciencedirect.com/science/article/ pii/S1876139909004393

13. Asociación Médica Mundial. Declaración de Helsinki de la AMM. Principios éticos para las investigaciones médicas en seres humanos [Internet]. Ferney-Voltaire: Asociación Médica Mundial; 1964 [citado 26 abr 2016]. Disponible en: https:// www.wma.net/es/policies-post/decla- racion-de-helsinki-de-la-amm-principios-eticos-para-las-investigaciones-medicas-en-seres-humanos/

14. Lolas F. Aspectos éticos de la investigación biomédica: Conceptos frecuentes en las normas escritas. Rev Med Chile. 2001; 129(6): 680-4.

15. Acevedo Pérez I. Aspectos éticos en la investigación científica. Cienc enferm. 2002; VIII(1): 15-8.

16. Ley $\mathrm{N}^{\circ} 20.120$, sobre investigación científica en el ser humano, su genoma, y prohíbe la clonación humana, Decreto 114 [Internet]. Santiago, Chile: Subsecretaría de Salud Pública, Ministerio de Salud; 2012 [citado 26 abril 2016]. Disponible en: http://www.leychile.cl/Navegar?idNor$\mathrm{ma}=1032919$ \&idVersion $=2012-05-19$

17. Riancho J, Maestre JM, Del Moral I, Riancho JA. Simulación clínica de alto realismo: una experiencia en el pregrado. Educ méd. [Internet]. 2012 Jun [citado 26 abr 2016]; 15(2): 109-115. Disponible en: http://dx.doi.org/10.4321/S157518132012000200009

18. Levett-Jones T, McCoy M, Lapkin S, Noble D, Hoffman K, Dempsey J, et al. The development and psychometric testing of the Satisfaction with Simulation Experience Scale. Nurse Educ Today. 2012; 31(7): 705-10.

19. Departamento de Enfermería y Fisioterapia. Universidad de Cádiz. Manual de casos clínicos simulados. Cádiz, España: Universidad de Cádiz; 2011/2012. p. 1338.

20. Jeffries P. A framework for designing, implementing, and evaluating simulations used as teaching strategies in nursing. Nurs Educ Perspect [Internet]. 2005 [citado 14 jun 2014]; 26: 96-103. Disponible en: https://www.ncbi.nlm.nih.gov/pubmed/15921126

21. Ambrosio AS. Formación de equipos de trabajo. Relaciones interpersonales: Transdoc [Internet]. Guatemala: Transdoc S.A.; 2013 [citado 13 ago 2017]. 
Disponible en: https://gt.transdoc.com/ articulos/recursos-humanos/Formacion-de-equipos-de-trabajo--Relaciones-interpersonales-/22954

22. Alconero A, Martínez A, Maestre J, García S, Cobo J, Moral I. La simulación clínica como instrumento de aprendizaje en cuidados cardiológicos: experiencia en el pregrado. Rev Esp Cardiol [Internet]. 2013 [citado 10 jun 2014]; 66(sup 1): 1133-1140.

23. Valencia C, Uribe A, Delgado J, Hernández M, Mercado C, Rubio L, et al. Estandarización de procedimientos de Enfermería y producción de videos como estrategia de innovación académica. Rev. Educ Cienc Salud [Internet]. 2013 [citado 20 may 2014]; 10 (Supl): 174-75. Dis- ponible en: http://www2.udec.cl/ofem/ recs/anteriores/vol10sup2013/RECS10sup2013.pdf

24. Bastías N, Bustamante C, Campos I, Ortiz L, Fasce E, Matus O, et al. Positiva percepción de los estudiantes frente a estrategia de simulación. Rev. Educ Cienc Salud [Internet]. 2014 [citado 20 may 2014]; 10(2): 252. Disponible en: http:// www2.udec.cl/ofem/recs/anteriores/vol10sup2013/RECS10sup2013.pdf

25. Achury DM, Achury LF, Rueda MJ. Satisfacción de los estudiantes de cuidado crítico con el uso de la simulación clínica en el proceso de enseñanza aprendizaje. CONNECT: The World of Critical Care Nursing. 2012; 9(1): 35-38. 